M. KONISHI

KŌDAI MATH. SEM. REP.

26 (1975), 194-200

\title{
ON MANIFOLDS WITH SASAKIAN 3-STRUCTURE OVER QUATERNION KAEHLER MANIFOLDS
}

\author{
Dedicated to Professor Yūsaku Komatu \\ on his sixtieth birthday
}

\author{
BY MARIKo KONISHI
}

$\S 1$. Introduction. Any complete Riemannian manifold admitting a regular $K$-contact 3 -structure is a $S^{3}(1)$ or $R P^{3}$-principal bundle over an almost quaternion manifold, where $S^{3}(1)$ denotes a sphere of curvature 1 and $R P^{3}=S^{3}(1) /\{ \pm I\}$ (See Tanno [7]). If the contact 3-structure is Sasakian, then the manifold is Einstein space and the base space becomes a quaternion Kaehler manifold with positive scalar curvature.

On the other hand, every quaternion Kaehler manifold $M$ admits a principal bundle $P$ over it, whose structure group is $S O(3)$ (Sakamoto [6]). In this note, we construct in $P, 3$-structure which is canonically associated with a given quaternion Kaehler structure. That is, we shall prove Theorem 2 in $\S 4$, which is corresponding to the theorem for a compact Hodge manifold, i. e.,

THEOREM 1. Let $M$ be a compact Hodge manifold. Then there exists a circle bundle over $M$, which admits a normal contact metric structure (Hatakeyama [1]).

\section{§2. Sasakian 3-structure.}

Let $(\tilde{M}, \tilde{g})$ be a Riemannian manifold and $\xi$ be a unit Killing vector. Define a tensor field of type (1.1) by

$$
\phi=\tilde{\nabla} \xi,
$$

where $\tilde{\nabla}$ denotes the Riemannian connection. Then we call $\xi$ a $K$-contact structure if $\phi$ satisfies

$$
\phi^{2}=-I+\alpha \otimes \xi,
$$

$\alpha$ being a 1 -form defined by $\alpha(\tilde{X})=\tilde{g}(\xi, \tilde{X})$. Next we denote by $N$ the Nijenhuis tensor of $\phi$ and by $\Phi$ the 2 -form defined by $\Phi(\tilde{X}, \tilde{Y})=\tilde{g}(\phi \tilde{X}, \tilde{Y})$. If the tensor

$$
S=N+2 \Phi \otimes \xi
$$

Received July 12, 1973. 
vanishes, we call $\xi$ a Sasakian structure.

Next we consider a set of mutually othogonal unit Killing vectors $\{\xi, \eta, \zeta\}$ satisfying

$$
[\xi, \eta]=2 \zeta, \quad[\eta, \zeta]=2 \xi, \quad[\zeta, \xi]=2 \eta,
$$

which is called a triple of Killing vectors. We put

and

$$
\phi=\tilde{\nabla} \xi, \quad \phi=\tilde{\nabla} \eta, \quad \theta=\tilde{\nabla} \zeta
$$

$$
\alpha(\tilde{X})=\tilde{g}(\xi, \tilde{X}), \quad \beta(\tilde{X})=\tilde{g}(\eta, \tilde{X}), \quad \gamma(\tilde{X})=g(\zeta, \tilde{X}) .
$$

If each of $\xi, \eta$ and $\zeta$ is a $K$-contact structure and satisfies

$$
\begin{array}{ccc}
\phi \phi=\theta+\alpha \otimes \eta, & \theta \phi=\phi+\beta \otimes \zeta, & \phi \theta=\phi+\gamma \otimes \xi, \\
\phi \phi=-\theta+\beta \otimes \xi, & \phi \theta=-\phi+\gamma \otimes \eta, & \theta \phi=-\phi+\alpha \otimes \zeta,
\end{array}
$$

then $\{\xi, \eta, \zeta\}$ is called a $K$-contact 3 -structure. For a $K$-contact 3 -structure, if each of $\xi, \eta$ and $\zeta$ is a Sasakian structure, then $\{\xi, \eta, \zeta\}$ is called a Sasakıan 3-structure.

\section{§3. Quaternion Kaehler manifold (See Ishihara [3]).}

Let $M$ be a differentiable manifold of dimension $n(=4 m)$. Assume that there is a 3-dimensional vector bundle $V$ consisting of tensors of type $(1,1)$ over $M$ satisfying the following condition.

a) In any coordinate neighborhood $U$ of $M$, there is a local base $\{F, G, H\}$ of $V$ such that

$$
\begin{gathered}
F^{2}=-I, \quad G^{2}=-I, \quad H^{2}=-I, \\
H G=-G H=F, \quad F H=-H F=G, \quad G F=-F G=H,
\end{gathered}
$$

$I$ denoting the identity tensor field of type (1.1) in $M$. Then the bundle $V$ is called an almost quaternion structure in $M$ and $(M, V)$ an almost quaternion manrfold.

In an almost quaternion manifold $(M, V)$, we take two intersecting coordinate neighborhoods $U, U^{\prime}$, and local bases $\left\{F_{U}, G_{U}, H_{U}\right\},\left\{F_{U^{\prime}}, G_{U^{\prime}}, H_{U^{\prime}}\right\}$ satisfying (3.1) in $U, U^{\prime}$, respectively. Then they have relations in $U \cap U^{\prime}$ as

$$
\begin{aligned}
& F_{U^{\prime}}=s_{11} F_{U}+s_{12} G_{U}+s_{13} H_{U} \\
& G_{U^{\prime}}=s_{21} F_{U}+s_{22} G_{U}+s_{23} H_{U} \\
& H_{U^{\prime}}=s_{31} F_{U}+s_{32} G_{U}+s_{33} H_{U}
\end{aligned}
$$

where $s_{j i}(j, i=1,2,3)$ form an element $s_{U U^{\prime}}=\left(s_{j i}\right)$ of the special orthogonal group $S O(3)$ of dimension 3.

Let $P$ be the associated principal bundle of $V$. That is, $P$ is the bundle whose transition functions and structure group are the same as $V$, but whose 
fibre is $S O(3)$ (=the real projective space $R P^{3}$ of dimension 3 ). Then the Lie algebra ${ }_{30}(3)$ of the structure group of $P$ admits a base $\left\{e_{1}, e_{2}, e_{3}\right\}$ such that

$$
e_{1}=\left(\begin{array}{rrr}
0 & 0 & 0 \\
0 & 0 & 2 \\
0 & -2 & 0
\end{array}\right), \quad e_{2}=\left(\begin{array}{rrr}
0 & 0 & -2 \\
0 & 0 & 0 \\
2 & 0 & 0
\end{array}\right), \quad e_{3}=\left(\begin{array}{rrr}
0 & -2 & 0 \\
2 & 0 & 0 \\
0 & 0 & 0
\end{array}\right)
$$

and hence they satisfy

$$
\left[e_{1}, e_{2}\right]=2 e_{3}, \quad\left[e_{2}, e_{3}\right]=2 e_{1}, \quad\left[e_{3}, e_{1}\right]=2 e_{2} .
$$

In any almost quaternion manifold $(M, V)$, there is a Riemannian metric such that

$$
\begin{gathered}
g\left(F_{U} X, Y\right)+g\left(X, F_{U} Y\right)=0, \quad g\left(G_{U} X, Y\right)+g\left(X, G_{U} Y\right)=0, \\
g\left(H_{U} X, Y\right)+g\left(X, H_{U} Y\right)=0
\end{gathered}
$$

hold for any local base $\left\{F_{U}, G_{U}, H_{U}\right\}$ and any vector fields $X, Y$. Assume that the Riemannian connection $\nabla$ of $(M, g)$ satisfies for any local base $\left\{F_{U}, G_{U}, H_{U}\right\}$

$$
\begin{aligned}
& \nabla_{X} F_{U}=\quad-2 r_{U}(X) G_{U}-2 q_{U}(X) H_{U} \\
& \nabla_{X} G_{U}=2 r_{U}(X) F_{U}+2 p_{U}(X) H_{U} \\
& \nabla_{X} H_{U}=2 q_{U}(X) F_{U}-2 p_{U}(X) G_{U}
\end{aligned}
$$

where $p_{U}, q_{U}$ and $r_{U}$ are certain 1 -forms defined in $U$. Then $(M, g, V)$ is called a quaternion Kaehler manifold and $(g, V)$ a quaternion Kaehler structure.

For each neighborhood $U$ in a quaternion Kaehler manifold $(M, V)$, we define a $\mathfrak{s}(3)$-valued 1 -form on $U$ by

$$
\omega_{U}=p_{U} e_{1}+q_{U} e_{2}+r_{U} e_{3} .
$$

Then, by virtue of (3.2) and (3.3), in the intersection of neighborhoods $U$ and $U^{\prime}$, we find

$$
\omega_{U^{\prime}}(X)=a d\left(s_{U U^{\prime}}{ }^{-1}\right) \cdot \omega_{U}(X)+\left(s_{U U^{\prime}}\right)_{*}(X) \cdot s_{U U^{\prime}}{ }^{-1}
$$

for every vector field $X$ on $P$, where ad denotes the adjoint representation of $S O(3)$ in $S_{0}(3)$, and $\left(s_{U U^{\prime}}\right)_{*}$ denotes the differential of the mapping $s_{U U^{\prime}}: U \cap U^{\prime}$ $\rightarrow S O(3)$. Hence there exists a connection form $\omega$ on $P$ such that

$$
\tau^{*} \omega=\omega_{U}
$$

where $\tau$ is a certain local cross-section of $P$ over $U$ (for detail, see p. 66 in Kobayashi-Nomizu [4]).

We denote by $\Omega$ the curvature form defined by the connection $\omega$. Then $\Omega$ is the $\mathfrak{B D}_{0}(3)$-valued 2 -form expressed by

$$
\Omega(\tilde{X}, \tilde{Y})=d \omega(\tilde{X}, \tilde{Y})+\frac{1}{2}[\omega(\tilde{X}), \omega(\tilde{Y})]
$$


where $\hat{X}, \tilde{Y}$ are vector fields in $P$ and $[$,$] denotes the bracket operation in$ $\xi_{\mathfrak{D}}(3)$. Then we have

$$
\begin{aligned}
\tau^{*} \Omega=\left(d p_{U}+q_{U} \wedge r_{U}\right) e_{1} & +\left(d q_{U}+r_{U} \wedge p_{U}\right) e_{2} \\
& +\left(d r_{U}+p_{U} \wedge q_{U}\right) e_{3}
\end{aligned}
$$

for each cross section $\tau: U \rightarrow P$ and 1-forms $p_{U}, q_{U}, r_{U}$ on $U$.

On the other hand, since any quaternion Kaehler manifold is an Einstein space (See Theorem 3.3 in Ishihara [3]), we have following relations

$$
\begin{gathered}
d p_{U}+q_{U} \wedge r_{U}=c A_{U}, \quad d q_{U}+r_{U} \wedge p_{U}=c B_{U}, \\
d r+p_{U} \wedge q_{U}=c C_{U},
\end{gathered}
$$

where $4 m(m+2) c$ is a constant equal to the scalar curvature of $(M, g)$, and $A_{U}(X, Y)=g\left(F_{U} X, Y\right), B_{U}(X, Y)=g\left(G_{U} X, Y\right), C_{U}(X, Y)=g\left(H_{U} X, Y\right)$.

\section{$\S 4$. Construction of Sasakian 3-structure.}

Let $(M, g)$ be a quaternion Kaehler manifold of dimension $n=4 m$, and $P$ be the associated $R P^{3}$-principal bundle over $M$. We denote by $\omega=\sum_{\imath=1}^{3} \omega_{i} e_{\imath}$ the infinitesimal connection in $P$ defined in the previous section. We define a pseudoRiemannian metric $g$ in $P$ by

$$
\tilde{g}=c \pi_{*} g+\sum_{i=1}^{3} \omega_{i} \otimes \omega_{i}
$$

where $c$ is the constant appearing in (3.6). If the scalar curvature of $M$ is positive, then $g$ is a Riemannian metric and if negative, $g$ is a pseudo-Riemannian metric of signature $(3, n)$. In both cases, $(M, g)$ is necessarily irreducible (Ishihara [3]).

We put

$$
\omega_{1}=\alpha, \quad \omega_{2}=\beta, \quad \omega_{3}=\gamma,
$$

then $\alpha, \beta$ and $\gamma$ are 1 -forms in $P$. Let $\xi, \eta, \zeta$ be fundamental vector fields corresponding to $e_{1}, e_{2}, e_{3}$, respectively. Then we have from (3.3)

and

$$
[\xi, \eta]=2 \zeta, \quad[\eta, \zeta]=2 \xi, \quad[\zeta, \xi]=2 \eta,
$$

Hence we have

$$
\begin{array}{lll}
\alpha(\xi)=1, & \alpha(\eta)=0, & \alpha(\zeta)=0, \\
\beta(\xi)=0, & \beta(\eta)=1, & \beta(\zeta)=0, \\
\gamma(\xi)=0, & \gamma(\eta)=0, & \gamma(\zeta)=1 .
\end{array}
$$

Proposition 1. In the associated principal bundle $P$ over a quaternion Kaehler manifold, there exists a triple of Killing vectors $\{\xi, \eta, \zeta\}$ with respect to 
the metric defined by (4.1), i.e. $\xi, \eta$ and $\zeta$ are mutually orthogonal unit Killing vectors satisfying

$$
[\xi, \eta]=2 \zeta, \quad[\eta, \zeta]=2 \xi, \quad[\zeta, \xi]=2 \eta,
$$

Proof. It remains to prove that $\xi, \eta, \zeta$ are all Killing vectors with respect to $\tilde{g}$ in (4.1). This is clear from the fact that $\sum_{i=1}^{3} \omega_{\imath} \otimes \omega_{i}$ is invariant under the action of $S O(3)$.

PROPOSITION 2. The triple of Killing vectors $\{\xi, \eta, \zeta\}$ defined in proposition 1 is a K-contact 3-structure, if $c>0$.

Proof. We define

$$
\phi=\tilde{\nabla} \xi, \quad \phi=\tilde{\nabla} \eta, \quad \theta=\tilde{\nabla} \zeta,
$$

$\tilde{\nabla}$ being Riemannian connection formed with $\tilde{g}$. Then we have

$$
\begin{gathered}
\phi \xi=0, \quad \phi \eta=0, \quad \theta \zeta=0, \\
\theta \eta=-\phi \zeta=\xi, \quad \phi \zeta=-\theta \xi=\eta, \quad \phi \xi=-\phi \eta=\zeta,
\end{gathered}
$$

since $\xi, \eta$ and $\zeta$ are mutually orthogonal unit vectors. Denoting by $T_{\tilde{p}}{ }^{\nu}(P)$ the the tangent space of a fibre at $\tilde{p} \in P$ and by $T_{\tilde{p}}^{H}(P)$ its orthogonal complemented space in $T_{\tilde{p}}(P)$, we see from (4.4) that $T_{\tilde{p}}{ }^{V}(P)$ and $T_{\tilde{p}}{ }^{H}(P)$ are invariant under the actions of the linear endomorphisms $\phi, \phi$ acd $\theta$ of $T_{\tilde{p}}(P)$. Hence we can put

$$
\begin{gathered}
\phi=\phi^{H}+\gamma \otimes \eta-\beta \otimes \zeta, \quad \phi=\phi^{H}+\alpha \otimes \zeta-\gamma \otimes \xi, \\
\theta=\theta^{H}+\beta \otimes \xi-\alpha \otimes \eta,
\end{gathered}
$$

where $\phi^{H}, \psi^{H}$ and $\theta^{H}$ denote the restricted actions of $\phi, \phi$ and $\theta$ on $T_{\tilde{p}}^{H}(P)$ for each $\tilde{p}$.

On the other hand, taking account of (3.5) (3.7), for each neighborhood $U$ in $M$ and a local cross section $\tau: U \rightarrow P$, we have

$$
\begin{aligned}
& (d \alpha-\beta \wedge \gamma)\left(\tau_{*} X, \tau_{*} Y\right)=c A_{U}(X, Y), \\
& (d \beta-\gamma \wedge \alpha)\left(\tau_{*} X, \tau_{*} Y\right)=c B_{U}(X, Y), \\
& (d \gamma-\alpha \wedge \beta)\left(\tau_{*} X, \tau_{*} Y\right)=c C_{U}(X, Y),
\end{aligned}
$$

$\tau_{*}$ denoting the differential of $\tau$. Since the curvature form is horizontal, we have

$$
\begin{aligned}
& (\phi-\gamma \otimes \eta+\beta \otimes \zeta)\left(\tau_{*} X\right)=\left(\tau_{*} F_{U} X\right)^{H}, \\
& (\phi-\alpha \otimes \zeta+\gamma \otimes \xi)\left(\tau_{*} X\right)=\left(\tau_{*} G_{U} X\right)^{H}, \\
& (\theta-\beta \otimes \xi+\alpha \otimes \eta)\left(\tau_{*} X\right)=\left(\tau_{*} H_{U} X\right)^{H},
\end{aligned}
$$

i. e. 


$$
\begin{aligned}
& \phi\left(\tau_{*} X\right)=\left(\tau_{*} F_{U} X\right)^{H}+\gamma\left(\tau_{*} X\right) \eta-\beta\left(\tau_{*} X\right) \zeta, \\
& \phi\left(\tau_{*} X\right)=\left(\tau_{*} G_{U} X\right)^{H}+\alpha\left(\tau_{*} X\right) \zeta-\gamma\left(\tau_{*} X\right) \xi, \\
& \theta\left(\tau_{*} X\right)=\left(\tau_{*} H_{U} X\right)^{H}+\beta\left(\tau_{*} X\right) \xi-\alpha\left(\tau_{*} X\right) \eta,
\end{aligned}
$$

where $\left(\tau_{*} F_{U} X\right)^{H}$ denotes the projection of $\tau_{*} F_{U} X$ to $T_{\tau(p)}{ }^{H}(P)$. Next we show that $\phi, \phi$ and $\theta$ satisfy (2.1) and (2.3). From (4.4) and (4.5) we have

$$
\begin{aligned}
\phi^{2}\left(\tau_{*} X\right) & =\phi\left(\left(\tau_{*} F_{U} X\right)^{H}+\gamma\left(\tau_{*} X\right) \eta-\beta\left(\tau_{*} X\right) \zeta\right) \\
& =\left(\tau_{*} F_{U}{ }^{2} X\right)^{H}-\gamma\left(\tau_{*} X\right) \zeta-\beta\left(\tau_{*} X\right) \eta \\
& =-\left(\tau_{*} X\right)^{H}-\beta\left(\tau_{*} X\right) \eta-\gamma\left(\tau_{*} X\right) \zeta \\
& =-\tau_{*} X+\alpha\left(\tau_{*} X\right) \xi .
\end{aligned}
$$

and

$$
\begin{aligned}
\phi\left(\phi\left(\tau_{*} X\right)\right) & =\phi\left(\left(\tau_{*} G_{U} X\right)^{H}+\alpha\left(\tau_{*} X\right) \zeta-\gamma\left(\tau_{*} X\right) \xi\right) \\
& =\left(\tau_{*} F_{U} G_{U} X\right)^{H}+\alpha\left(\tau_{*} X\right) \eta \\
& =-\left(\tau_{*} H_{U} X\right)^{H}+\alpha\left(\tau_{*} X\right) \eta \\
& =-\theta\left(\tau_{*} X\right)+\beta\left(\tau_{*} X\right) \xi \\
\phi\left(\phi\left(\tau_{*} X\right)\right) & =\phi\left(\left(\tau_{*} F_{U} X\right)^{H}+\gamma\left(\tau_{*} X\right) \eta-\beta\left(\tau_{*} X\right) \zeta\right) \\
& =\left(\tau_{*} G_{U} F_{U} X\right)^{H}+\beta\left(\tau_{*} X\right) \xi \\
& =\left(\tau_{*} H_{U} X\right)^{H}+\beta\left(\tau_{*} X\right) \xi \\
& =\theta\left(\tau_{*} X\right)+\alpha\left(\tau_{*} X\right) \eta
\end{aligned}
$$

because of (3.1). Similarly we obtain the other relations in (2.3). That is, $\{\xi, \eta$, $\zeta\}$ defines a $K$-contact 3 -structure.

Going through the process of having induced quaternion (Kaehler) structure from regular $K$-contact (Sasakian) 3-structure (cf. Ishihara [2] and Konishi [5]), our construction of $K$-contact 3 -structure $\{\xi, \eta, \zeta\}$ is quite natural. That is to say, we have obtained a fibred Riemannian space $(P, \tilde{g})$ with $K$-contact 3 -structure $\{\xi, \eta, \zeta\}$ which induces the given quaternion Kaehler structure in the base space. As shown in [5], such a $K$-contact 3 -structure is necessarily a Sasakian 3-structure. Thus we have

THEOREM 2. Let $M$ be a quaternion Kaehler manifold of dimension $n=4 m$. Then there exists a canonically associated $R P^{3}$-principal bundle $P$ over $M$. If the scalar curvature of $M$ is positıve, $P$ admits a Sasakıan 3-structure and if negative, the induced metric by (4.1) has signature $(3, n)$. 


\section{BIBLIOGRAPHY}

[1] Y. Hatakeyama, Some notes on differentiable manifolds with almost contact structures, Tôhoku Math. J., 15 (1963), 176-181.

[2] S. Ishihara, Quaternion Kaehler manifolds and fibred Riemannian spaces with Sasakian 3-structure, Kōdai Math. Sem. Rep., 25 (1973), 321-329.

[ 3 ] S. Ishinara, Quaternion Kaehler manifold, J. Diff. Geom., 9 (1974), 483-500.

[4] S. Kobayashi And K. Nomizu, Foundations of differential Geometry, vol. I, Interscience tract No. 15, (1963).

[55:] M. Konishi, Note on regular $K$-contact structures, Ochanomizu Nat. Sci. Rep., 24 (1973), 1-5.

[6] K. SaKamoto, On the topology of quaternionic Kaehler manifolds, Tôhoku Math. J., 26 (1974), 389-406.

[.7] S. TANno, Killing vectors on contact Riemannian manifolds and fiberings related to the Hopf fibrations, Tôhoku Math. J., 23 (1971), 313-334.

Department of Mathematics,

Tokyo Institute of Technology. 